\title{
New Distance Estimates to RR Lyrae Stars: Towards a Definitive Luminosity Calibration
}

\author{
Andrew Layden \\ Bowling Green State University, USA, e-mail: layden@baade.bgsu.edu
}

\begin{abstract}
We describe two projects currently underway to provide an improved calibration of RR Lyrae luminosities.
\end{abstract}

\section{Introduction}

For over half a century, RR Lyrae variables (RRL) have played a vital role in determining the Population II distance scale. They yield distances to globular clusters, to the Galactic center, and to nearby galaxies such as the LMC and the Sagittarius dwarf. When combined with photometry of main sequence turn-off (MSTO) stars and theoretical isochrones, the globular cluster distances yield ages, providing us with one of the most precise clocks for investigating the formation and evolution of the Galaxy. Finally, the ages of the oldest globulars in our Galaxy place a firm lower limit on the age of the Universe, and thus provide an important constraint on cosmological models.

Despite the importance of RRL, astronomers have been unable to reach a consensus on the luminosity of these standard candles. Recent reviews by Chaboyer (1998) and Carretta et al. (2000) discuss the results of various methods used to obtain the RRL luminosity, and assess the methods' strengths and weaknesses. These methods include trigonometric parallaxes from HIPPARCOS, statistical parallaxes using HIPPARCOS proper motions, main sequence fits to subdwarfs with HIPPARCOS parallaxes, etc. The results of the individual methods span a quarter magnitude in absolute magnitude. The mean of the six methods discussed by Chaboyer $(1998)$ is $M_{V}(R R)=0.56$ at $[\mathrm{Fe} / \mathrm{H}]=-1.6$, with a standard deviation of 0.12 mag. However, the distribution of the six values is non-Gaussian, suggesting the presence of systematic errors in addition to random errors in some or all of the methods. Such disagreements have led some authors to suggest that field and cluster RRL may exhibit different $M_{V}(R R)-[F e / H]$ relations (e.g., Gratton, 1998).

The quoted uncertainties in the calibrations of Chaboyer (1998) and Carretta et al. (2000) are $\sim 0.12 \mathrm{mag}$. This level of uncertainty yields distance uncertainties of $5 \%$, and translates into $\sim 10 \%$ uncertainty in age estimates. When combined with uncertainties in composition, physics of the models, etc., the total uncertainty in absolute age estimates is larger still. Ideally, we would like to get the total age uncertainty below $5 \%$ in order to provide the clearest constraints on the formation history of the Galaxy and on cosmological models. To do this, we must know $M_{V}(R R)$ to $\sim 0.03 \mathrm{mag}$ or less. 


\section{Method 1: White dwarf sequence fitting in M5}

A new technique for measuring globular cluster distances has become available in recent years: fitting the photometric sequence of white dwarfs (WDs) to field WDs of known absolute magnitude or to WD model sequences (Renzini et al., 1996). There are several advantages to this method. (1) WD physics is simpler than MSTO physics, since there is no convection and the atmospheric opacities are dominated by $\mathrm{H}, \mathrm{He}$. (2) WD luminosity is insensitive to the star's original composition, so that WDs in low metallicity clusters may be compared directly with nearby disk WDs (originally solar metallicity). The WD fitting method thus provides a welcome complement to the techniques discussed in Sect. 1.

Of course, disadvantages exist with the WD fitting method. (1) WD luminosities are sensitive to the WD mass, so care must be exercised to select nearby WDs whose mass matches those of the WDs in globulars. (2) WDs in globulars are faint: typically $>10$ mag below the level of the HB.

Well delineated WD sequences have been detected in several globulars (NGC 6752, Renzini et al., 1996; NGC 6397, Cool et al., 1996; M4, Richer et al., 1997; 47 Tuc, Zoccali et al., 2000). Of these clusters, M4 is the only one with a significant RRL population, and M4 suffers from strong, variable reddening with a non-standard reddening law. M5 (NGC 5904) is the nearest, low-reddening, RRL-rich cluster with which to obtain a high quality WD-based estimate of $M_{V}(R R)$.

Using the Hubble Space Telescope (HST), our team (A. Layden [PI], A. Cool, T. von Hippel, \& A. Sarajedini) has obtained 15 orbits of deep photometry in $\mathrm{F} 555 \mathrm{~W}$ and $\mathrm{F} 814 \mathrm{~W}(V$ and $I)$ in one field in M5. Our photometric reduction procedure is to find all sources using Source Extractor (Bertin \& Arnouts, 1996), obtain photometry of each object in this source list using DAOPHOT II and Allframe (Stetson, 1994), and then to cull out non-stellar objects using the classification provided by SOURCE EXTRACTOR.

Our work so far has focused on the WF2 chip. We have obtained a CMD reaching from the MSTO at $V \approx 18 \mathrm{mag}$ to $V_{\text {lim }} \approx 28 \mathrm{mag}$. The WD sequence begins at $V \approx 25 \mathrm{mag}$, giving us several mag of the WD sequence to perform the fitting. However, there is significant contamination from background objects, presumably field halo stars and unresolved galaxies. We are continuing to experiment with the parameters used in Source ExTraCtor and Allframe to improve the depth, completeness, and quality of the photometry of the WDs. Once we have settled on the optimal parameters, we will process the WF3 and WF4 chips.

We are hopeful that the existing photometry will yield a tight WD sequence, but we are considering the possibility of obtaining additional HST images. Not only would this improve the photometric signal-to-noise, but a second epoch would provide differential astrometry that would help us to identify non-cluster objects and to clean the WD region of the CMD. In summary, the WD fitting technique is promising, but the faintness of the WDs makes it an extreme technical challenge. This technique may yield results for additional globulars when data are acquired with adaptive optics instruments on large ground-based telescopes, or with the Next Generation Space Telescope. 


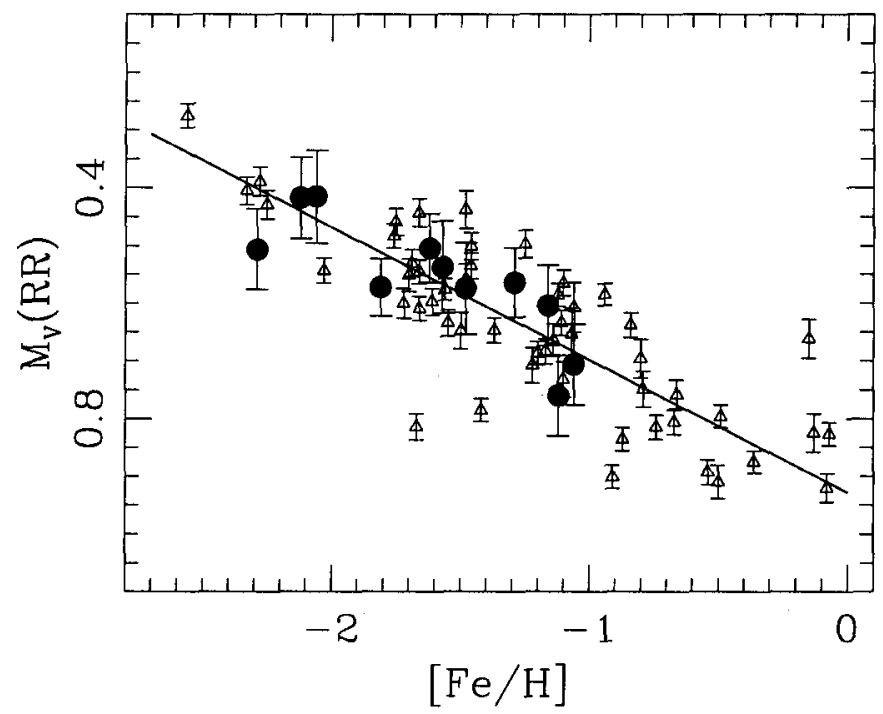

Figure 1. Simulated results of SIM RRL absolute magnitudes as a function of metallicity. See Sect. 3 for a description of the symbols and modeling technique.

\section{Method 2: Space-based trigonometric parallaxes with SIM}

NASA's Space Interferometry Mission (SIM) is a satellite-based white light interferometer with a 10-m baseline whose goal is to produce parallaxes accurate to $4 \mu$-arcsec. Unlike the all-sky astrometry of HIPPARCOS, FAME, and GAIA, SIM operates in pointed mode, enabling it to measure finer parallaxes on fainter objects $\left(V_{\text {lim }} \approx 20 \mathrm{mag}\right.$ ). This will enable it to measure direct distances to nearby globular clusters with errors of a few percent. Our team (B. Chaboyer [PI], B. Carney, D. Duncan, T. Girard, D. Latham, A. Layden, A. McWilliam, A. Sarajedini, M. Shao) has been granted "key project" status and $\sim 800$ hours of satellite time to study the distances of 21 nearby globulars, along with 60 field RRL and another 60 field MSTO and subgiant stars. Our primary goals are to determine the ages of the oldest globulars to $<5 \%$ and thus constrain the age of the Universe, and to determine the relative ages of clusters over a range in $[\mathrm{Fe} / \mathrm{H}]$ to determine the star formation and chemical evolution history of the early Galaxy.

The globulars were chosen to span a wide range in $[\mathrm{Fe} / \mathrm{H}], \mathrm{HB}$ type, and Oosterhoff class. Eleven of the clusters are rich in RRL, and provide a unique opportunity to refine the $M_{V}(R R)-[F e / H]$ relation. The 60 field RRL were carefully selected to span the maximal range in both $[\mathrm{Fe} / \mathrm{H}]$ and evolutionary state (as proxied by pulsation period), and will provide an important check and complement on the $M_{V}(R R)-[F e / H]$ relation based on the globulars. 
Our simulations of the expected results are shown in Fig. 1, where each solid circle represents the mean $M_{V}$ of the RRL in one globular, and each triangle marks the $M_{V}$ of one field RRL in our candidate list. The error bars are estimated from the expected errors in parallax, reddening, and apparent magnitude for each star in our candidate list. The scatter of the field RRL is dominated by the cosmic scatter of $M_{V}$ at constant $[\mathrm{Fe} / \mathrm{H}]$ that we adopted for the simulation $(0.08 \mathrm{mag} \mathrm{rms})$. Monte Carlo simulations of 1000 trials suggest that we will determine both coefficients of the relation $M_{V}(R R)=c_{0}+c_{1}([\mathrm{Fe} / \mathrm{H}]+1.5)$ to $0.02-0.03$ mag. Clearly, this represents a vast improvement over the current calibrations discussed in Sect. 1. Also, comparison between the relations from field and cluster RRL will resolve the question of whether these populations share the same calibration (e.g., Gratton, 1998).

Currently, SIM is scheduled for launch in 2009. Preliminary results may be available mid-way through the 5 year mission, and final results should be available by 2015 . While this seems like far in the future, it is perhaps not too long to wait for a definitive answer to a debate that has raged for half a century.

\section{References}

Bertin, E. \& Arnouts, S. 1996, A\&AS, 117, 393

Carretta, E., et al. 2000, ApJ, 533, 215

Cool, A., Piotto, G., \& King, I. 1996, ApJ, 468, 655

Chaboyer, B. 1998, in Post-Hipparcos Cosmic Candles, eds. A. Heck \& F. Caputo, (Kluwer: Dordrecht), 111

Gratton, R. 1998, MNRAS, 296, 739

Renzini, A., et al. 1996, ApJ, 465, L23

Richer, H., et al. 1997, ApJ, 484, 741

Stetson, P. 1994, PASP, 106, 250

Zoccali, M. et al. 2000, ApJ, 553, 743

\section{Discussion}

McNamara: Using high-amplitude $\delta$ Scuti stars (HADS) as standard candles, I find an $M_{V}(R R)$ calibration that supports the "long" distance scale, but which has a cluster-to-cluster scatter ranging over $\sim 0.7$ mag. How do you reconcile this with the RRL-based calibrations?

Layden: Within a given cluster, RRL magnitudes scatter by 0.05 to $0.10 \mathrm{mag}$ (rms). Cluster-to-cluster, Carretta et al. (2000) found an rms scatter of $\sim 0.06$ mag around their $M_{V}(R R)-[F e / H]$ relation based on main sequence fits. Similarly small internal scatters are found using the other methods, suggesting that RRL are self-consistent standard candles, even if we do not yet know the correct calibration. It is also worth remembering that the HADS in globular clusters are all blue stragglers - presumably coalescenced close binaries. It is likely that each globular-aged HADS has undergone unique and significant mixing, making them a very heterogeneous group of stars. 